\title{
Difference of auxin transport inhibitor treatment on several development processes between Arabidopsis and Nicotiana seedlings
}

\author{
Lijuan Zhou ${ }^{1}$, Erjuan Chen ${ }^{1}$, Shanna Chen ${ }^{1}$, Yougang Zhang ${ }^{2, *}$ (Corresponding author) \\ 1 School of Life Sciences, Yunnan University, Kunming, Yunnan Province, China \\ 2 School of Energy And Environment Science, Yunnan Normal University, Kunming, Yunnan Province, \\ China
}

Key words: auxin transport inhibitor; Arabidopsis; Nicotiana; development

Abstract. Auxin transport and signaling enforce stomatal patterning as well as the size of their stem cell compartment in Arabidopsis. But it remains unknown whether this regulation is also existed in other species. We use Nicotiana as an example to analysis the function of auxin transport inhibitor NAP and BFA on its stomatal developement on the epidermis of cotyledon taking Arabidopsis as control. Here we report that there is an inter-specific difference between Nicotiana and Arabidopsis under exogerous auxin inhibitors (NPA and BFA) treatment in stomatal development. No stomatal development defect can be detected in Nicotiana treated with NPA and BFA under the same concentration which can induce patterning and morphogenesis abnormality of stoma in Arabidopsis.

\section{INTRODUCTION}

Auxin widely regulates plant development, such as by coordinating the placement and patterning of organs and cells, most of this research were based on the model plant, Arabidopsis, for its small and completely sequenced genome[1,2,3,4,5,6,7]. In Nicotiana, auxin aslo regulate cell division, polarity formation, seed germination as well as stomata movement $[8,9,10]$. Recently Le et al reported auxin transport and signaling regulate stomatal patterning and development in Arabidopsis[11], But it remains unknown whether this regulation mechanism is also existed in other species. Use Nicotiana as subject, effect of auxin transport inhibitor NAP[12,13] and BFA[14,15] on its stomatal developement was analyzed to compare with Arabidopsis.

\section{MATERIAL AND METHODS}

\section{Plants and Growth Conditions}

All experiments described in this study involve Arabidopsis thaliana ecotype Columbia(col-0) and Nicotiana tabacum (K326). Arabidopsis thaliana and Nicotiana tabacum were grown at $24^{\circ} \mathrm{C}$ in $1 / 2 \mathrm{MS}$ agar plates in incubator with 12-hr-light/12-hr-dark cycles, and specific stages of rosette leaves were used.

\section{ChemicalTreatment}

Seedling were grown in three types culture plates; all obtained from Sigma-Aldrich NPA (Sigma 33371 ) and BFA (Beyotime S1536, Geldner et al., 2001) were dissolved in DMSO and used at final concentration of $20 \mu \mathrm{M}$, and as a control, the same volume of DMSO was added.

\section{Microscopy}

For observation by bright-field microscopy, seedling were analysed using an BSK41 compound light microscope(Olympus Instruments).

\section{Quantification}

For quantification of pavement cells and stomata, calculation of the stomata/stomatal index on the 4th day of the culture in Arabidopsis thaliana, and on the 6th day of the culture in Nicotiana tabacum. Calculating the germination rate every day from the 3rd to 5th day of the culture in Arabidopsis thaliana, from 5th to7th day in Nicotiana tabacum. Calculating the rate of gravitropism on 6th day of the culture in Arabidopsis thaliana, on 6th day of the culture in Nicotiana tabacum. 


\section{RESULT}

\section{Effect of NPA and BFA on Seed Germination Rate of Both Species}

For Arabidopsis, 20uM NPA and BFA treatment have seldom effect on their seed germination. But they do prolong the time needed for seed germination in Nicotiana. As showing in figure1, both kind of seeds are germinated mainly on 6days after planting on the MS medium. More than $84 \%$ of the seeds were germinated with or without NPA and BFA treatment in Arabidopsis, while only 19.24\% and $75.73 \%$ were germinated in Nicotiana with BFA and NPA treatment respectively. Compared with its untreated control, BFA dramatically decreased the seed germination rate of Nicotiana, which means its seed is more sensitive to BFA than that of Arabidopsis, and more sensitive to BFA than to NPA. Thus auxin polar transport during seed germination is essential to Nicotiana since the fungal toxin Brefeldin A disrupts PIN recycling, and thus auxin transport, by inhibiting ARF-GEF activity.
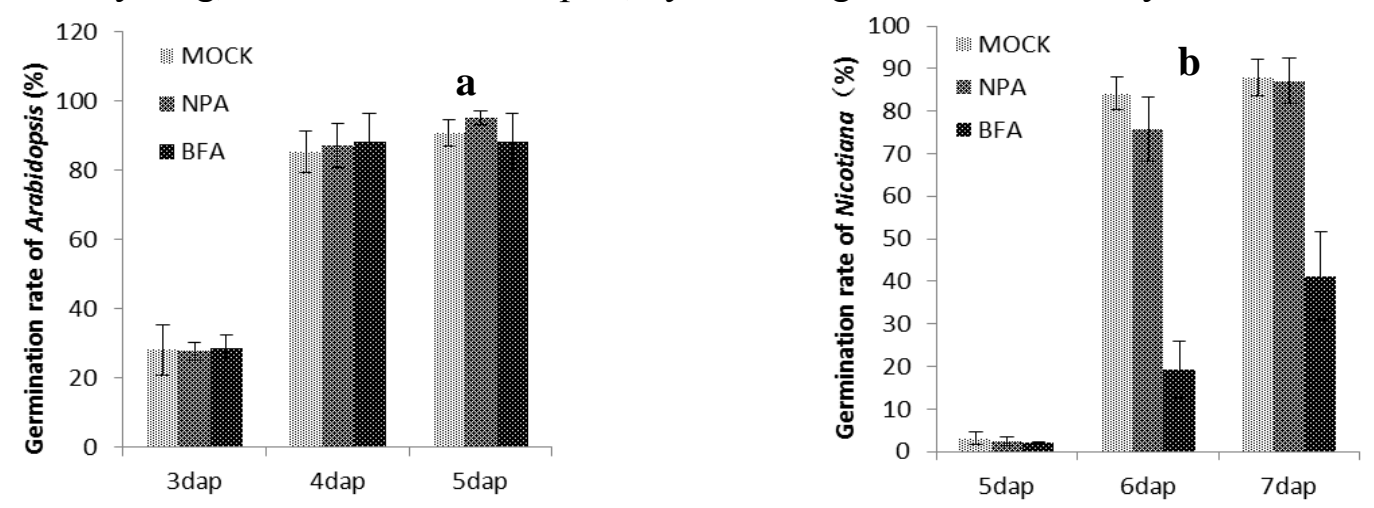

Figure 1. Effect of auxin ploar transport inhibitor on germination rate in Arabidopsis and Nicotiana（ $\mathbf{n}>\mathbf{1 0 0}$ )

(a) indicate germination rate of Arabidopsis; (b) indicate germination rate of Nicotiana.

\section{Effect of NPA and BFA on Root Development of Both Species}

In both species, defect of gravity tropism in 7days seedlings after planting were detected without obvious difference between two species. As showing in Figure2, BFA has more severe effect on this than NPA, both decrease intensively the rate of normal seedlings with down-forward roots grown on the vertical MS plates. Also, same inhibition effect on root elongation and lateral root formation were detected in both species seedlings.

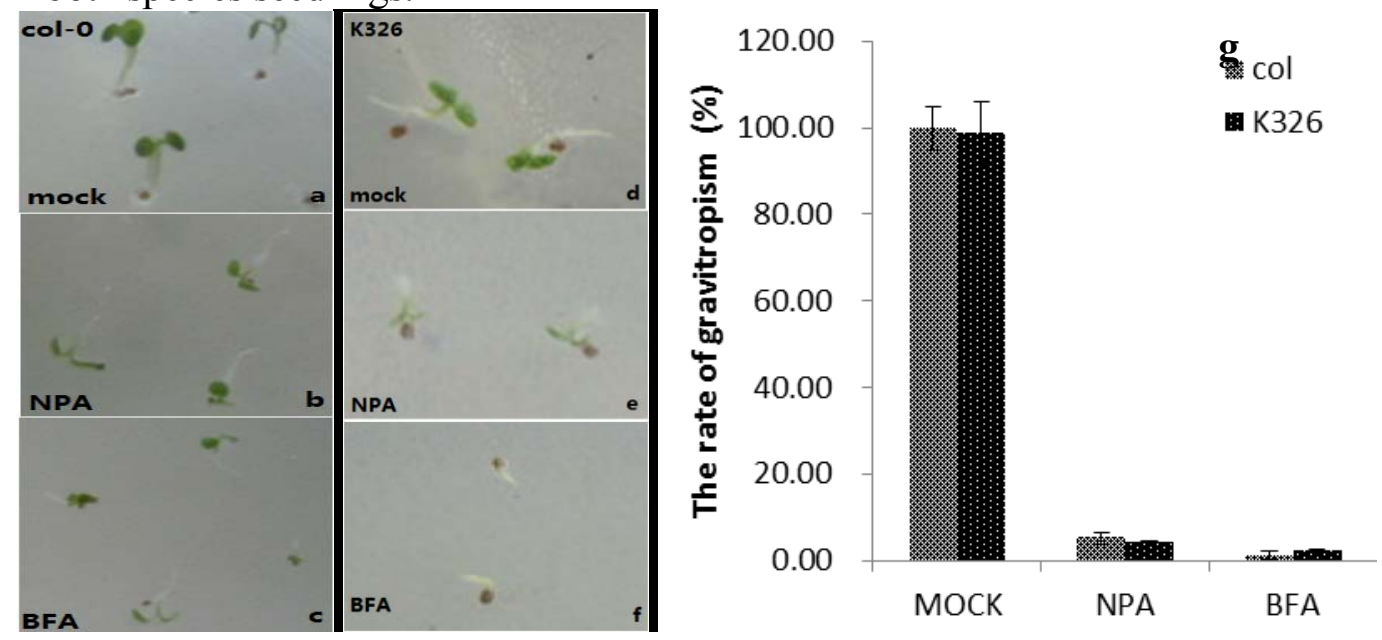

Figure 2. Effect of auxin ploar transport inhibitor on development of root in Arabidopsis and Nicotiana ( $\mathbf{n}>\mathbf{1 0 0}$ )

(a)indicate the mock sample of col-0; (b) indicate col-0 deal with NPA; (c)indicate the col-0 deal with BFA; (d) indicate the mock of K326; (e) indicate the K326 deal with NPA; (f) indicate the K326 deal with BFA; (g) indicate the rate of gravitropism in Arabidopsis and Nicotiana. 


\section{Effect of NPA and BFA on Stomatal Development of Both Species}

We then monitored the stomatal development on abaxial epidermis of cotyledon in different seedlings. Among the seedlings of Arabidopsis, up to $61.8 \%$ and $85.7 \%$ seedlings showed defect in stomata patterning or/and density after NPA and BFA treatment, while only $4.36 \%$ and $7.34 \%$ showed abnomal in Nicotinan.

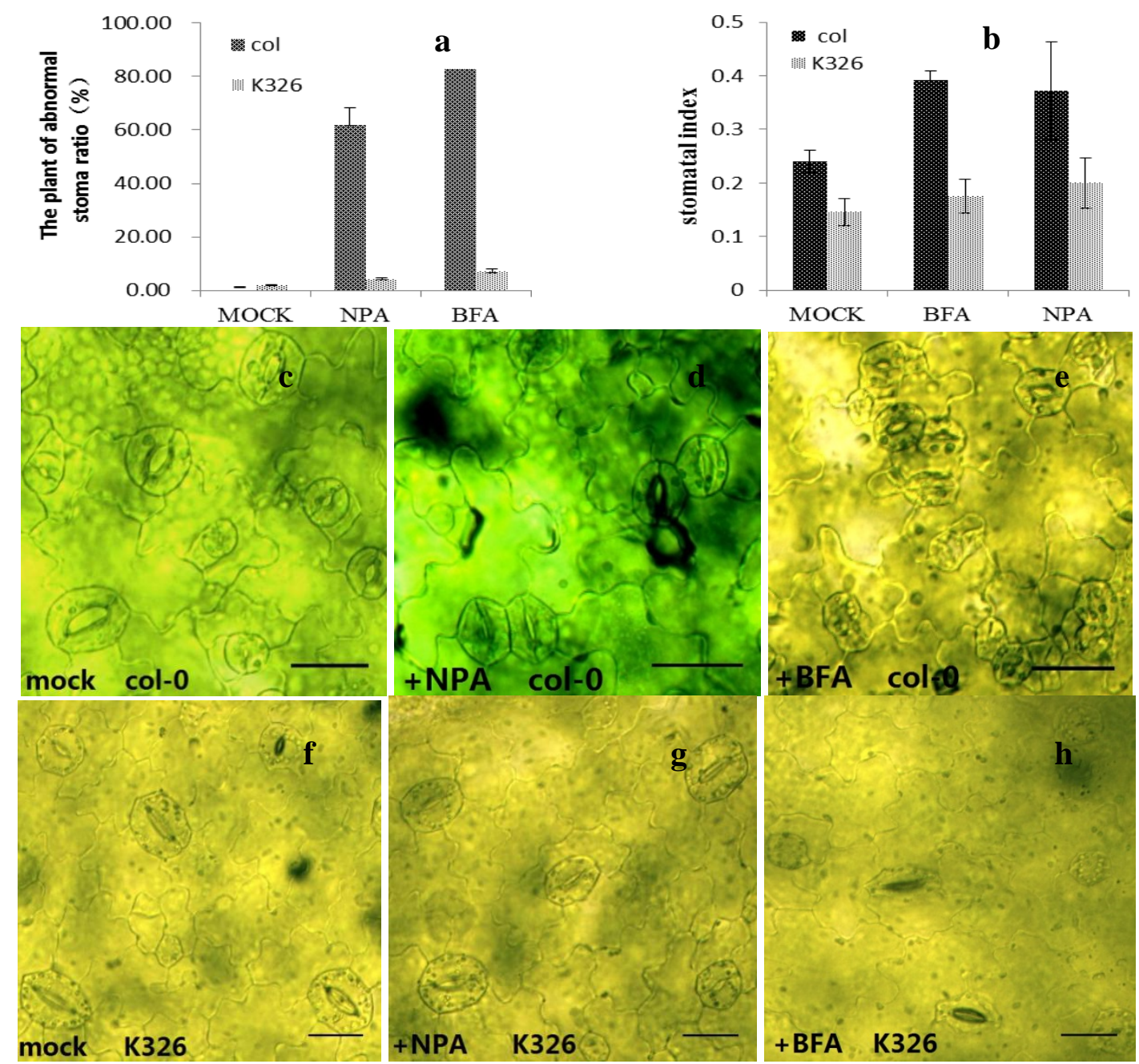

Figure 3. Effect of auxin ploar transport inhibitor on development of stoma in Arabidopsis and Nicotiana ( $\mathbf{n}>\mathbf{1 0 0})$

(a)indicate the plant of abnormal stomata ratio in Arabidopsis and Nicotiana; (b)indicate the stomatal index in Arabidopsis and Nicotiana;(c) indicate the mock of col-0; (d) indicate the col-0 deal with NPA;(e) indicate the col-0 deal with BFA;(f) indicate the mock of K326; (g)indicate the K326 deal with NPA;(h) indicate the K326 deal with NPA.

Stomata are separated by at least one intervening cell. NPA and BFA treatment induced sharp stomatal patterning defects as showing in figure3, stomatal clusters caused by one-cell-spacing interruption and enlarged stomata owing to morphogenesis defect were detected in chemical-treated Arabidopsis seedlings . On the contrary, none of such abnormalities were found in all the treated Nictoania seedlings with the same chemical concentration.

As for stomatal density, NPA induced a stomatal index (stomata number per $\mathrm{mm}^{2}$ epidermis area ) increase from 0.24 to 0.37 , and BFA induced a stomatal index (stomata number per $\mathrm{mm}^{2}$ epidermis area ) increase from 0.24 to 0.39 in Arabidopsis. While in Nictoania, the ratio only increase slightly 0.15 to 0.2 for NPA and 0.15 to 0.18 for BFA. 


\section{CONCLUSION}

As a major hormone, auxin controls almost every aspect of plant growth and development. Auxin transport, as well as signaling, involves in many aspect of development process like root elongation, lateral root formation, floral development and so on. Our results also proved auxin transport is essential for seed germination, root development and root gravity tropism in both Arabidopsis and Nicotiana. Auxin transport and signaling enforce stomatal patterning as well as the size of their stem cell compartment in Arabidopsis. But under the treatment of same concentration of inhibitors NPA and BFA, both species exhibited great difference in response in stomatal development, more in detail, the sensitivity to NPA and BFA of stomatal patterning and density differed. Nicotiana seems more resistance to NPA and BFA, thus to auxin transport inhibition.

What is the reason of this inter-specific difference need to be further studied, for differed sensitivity to chemicals or different stomatal patterning regulation mechanism in these two species may help us to understand more details about the stomatal development regulation mechanism based on more data from more species throughout the plant kingdom. For the former, Our results may remind researchers of the difference of sensitivity to chemical inhibitor in various species when they need to adopt exogenous treatment and draw their conclusion. For the latter, the possibility may exist, and if it so, more stomatal development regulation mechanism and comparison research will be made.

\section{REFERENCE}

[1] Benjamins R, Scheres B. 2008. "Auxin: the looping star in plant development”. Annu Rev Plant Biol.59: 443-465.

[2] Jennifer Normanly, Janet P. Slovin, Jerry D. 2010. "Auxin Biosynthesis and Metabolism". Plant Hormones. 36-62.

[3] Paque, S., Mouille, G., Grandont, L., et al. 2014. "AUXIN BINDING PROTEIN1 Links Cell Wall Remodeling, Auxin Signaling, and Cell Expansion in Arabidopsis". Plant Cell. 26: 280-295.

[4] Tromas, A., Braun, N., Muller, P., et al. 2009. "The AUXIN BINDING PROTEIN 1 is required for differential auxin responses mediating root growth". PLOS ONE, 4: e6648.

[5] Braun, N., Wyrzykowska, J., Muller, P., et al. 2008. "Conditional repression of AUXIN BINDING PROTEIN1 reveals that it coordinates cell division and cell expansion during postembryonic shoot development in Arabidopsis and tobacco". Plant Cell . 20: 2746-2762.

[6] Xu T, Wen M, Nagawa S, et al. 2010. "Cell surface- and rho GTPase based auxin signaling controls cellular interdigitation in Arabidopsis".Cell. 143:99-110.

[7] Xu T, Dai N, Chen J, et al. 2014. "Cell surface ABP1-TMK auxin-sensing complex activates ROP GTPase signaling”. Science. 343(6174): 1025-1028.

[8] Jan Petrášek, Miroslav Elčkner, David A. Morris, Eva Zažímalová. 2002. "Auxin efflux carrier activity and auxin accumulation regulate cell division and polarity in tobacco cells". Planta , 216(2):302-308

[9] Li Yan, LI Hong-Yan, WANG Qing, et al. "Role and Relationship of Oligochitosan, NO and Phytohormones in Stomatal Movement of Tobacco (Nicotiana tabacum L. cv. Samsun NN)". Plant Physiology Communications, 2010,46(6):575-578

[10]Li Zhen-hua. 2013. “Advances in Hormones During Tobacco Seed Germination”. Journal of Yunnan Agricultural University, 28 ( 3) : 416-423

[11] Le, J. Liu X-G, Yang K-Z, et al. 2014. Auxin transport and activity regulate stomatal patterning and development. Nat. Commun. 5:3090.

[12] Allan A C, Rubery P H. 1991. "Calcium deficiency and auxin transport in Cucurbita pepo L. seedlings". Planta. 183:604-612.

[13] Donaldson A W, Muday G K. 1993. "Auxin transport and root de-velopment in different plant species:Is there a correlation”. Plant Physiol, 102(s):323 .

[14] Steinmann. T., Geldner, N., Grebe, M. 1999. "Coordinated polar localization of auxin efflux carrier PIN1 by GNOM ARF GEF”. Science. 286:316-318. 